\title{
The exact asymptotic behavior of blow-up solutions to a highly degenerate elliptic problem
}

Ling $\mathrm{Mi}^{*}$

"Correspondence: mi-ling@163.com School of Science, Linyi University, Linyi, Shandong 276005, P.R. China

\begin{abstract}
In this paper, by constructing new comparison functions, we mainly study the boundary behavior of solutions to boundary blow-up elliptic problems for more general nonlinearities $f$ (which may be rapidly varying at infinity) $\Delta_{\infty} u=b(x) f(u)$, $x \in \Omega,\left.u\right|_{\partial \Omega}=+\infty$, where $\Omega$ is a bounded domain with smooth boundary in $\mathbb{R}^{N}$, and $b \in C(\bar{\Omega})$ which is positive in $\Omega$ and may be vanishing on the boundary and rapidly varying near the boundary.
\end{abstract}

Keywords: highly degenerate elliptic equations; blow-up solutions; the exact asymptotic behavior; comparison functions

\section{Introduction and the main results}

In this paper, we consider the exact asymptotic behavior of solutions to the following boundary blow-up elliptic problem:

$$
\triangle_{\infty} u=b(x) f(u), \quad u>0, x \in \Omega,\left.u\right|_{\partial \Omega}=\infty
$$

where the operator $\Delta_{\infty}$ is the $\infty$-Laplacian, a highly degenerate elliptic operator given by

$$
\triangle_{\infty} u:=\left\langle D^{2} u D u, D u\right\rangle=\sum_{i, j=1}^{N} D_{i} u D_{i j} u D_{j} u,
$$

$\Omega$ is a bounded domain with smooth boundary in $\mathbb{R}^{N}(N \geq 2), b$ satisfies

$\left(\mathrm{b}_{1}\right) \quad b \in C(\bar{\Omega})$ is positive in $\Omega$,

and $f$ satisfies

$\left(\mathrm{f}_{1}\right) f \in C^{1}([0, \infty)), f(0)=0, f$ is increasing on $(0, \infty)$;

$\left(\mathrm{f}_{2}\right) \int_{1}^{\infty} \frac{d v}{(f(v))^{\frac{1}{3}}}<\infty$

$\left(\mathrm{f}_{3}\right)$ there exists $C_{f}>0$ such that $\lim _{s \rightarrow+\infty} f^{\prime}(s) \int_{s}^{\infty} \frac{d v}{f(v)}=C_{f}$.

The $\infty$-Laplacian has been the subject of extensive investigation since the fundamental work of Aronsson [1] in which he established that the equation $\Delta_{\infty} u=0$ is the EulerLagrange equation for smooth absolute minimizers. As a result of the high degeneracy 
of the $\infty$-Laplacian, the associated Dirichlet problems may not have classical solutions. Therefore solutions are understood in the viscosity sense, a concept introduced by Crandall and Lions [2], Crandall et al. [3], and Crandall et al. [4], and to be defined in Section 2. Later, Jensen [5] proved the existence and uniqueness of the viscosity solutions to the Dirichlet problem to the infinity harmonic equation. Since then, the infinity Laplace equation has been attracting considerable attention and we direct the reader to see [6-8] and the references therein.

By a solution to the problem (1.1), we mean a nonnegative function $u \in C(\Omega)$ that satisfies the equation in the viscosity sense (see Section 2 for definition) and the boundary condition with $u(x) \rightarrow \infty$ as the distance function $d(x):=\operatorname{dist}(x, \Omega) \rightarrow 0$. Such a solution is called a boundary blow-up solution. Recently, A Mohammed and S Mohammed $[9,10]$ first supplied a necessary and sufficient condition

$$
\int_{a}^{\infty} \frac{d s}{\sqrt[4]{F(s)}}<\infty, \quad \forall a>0, F(s)=\int_{0}^{s} f(v) d v
$$

for the existence of solutions to problem (1.1).

The investigation of boundary blow-up problems for elliptic equations has a long history. Early studies mainly focused on problems involving the classical Laplace operator $\Delta$, i.e.

$$
\triangle u=b(x) f(u), \quad u>0, x \in \Omega,\left.u\right|_{\partial \Omega}=\infty,
$$

The problem (1.3) arises in Riemannian geometry, mathematical physics or population dynamics, and has been discussed and extended by many authors in many contexts; see, for instance, [11-28] and the references therein.

For $b \equiv 1$ on $\Omega$ and $f$ satisfying $\left(\mathrm{f}_{1}\right)$, Keller and Osserman [21, 26] first supplied the necessary and sufficient condition

$$
\int_{a}^{\infty} \frac{d s}{\sqrt{2 F(s)}}<\infty, \quad \forall a>0, F(s)=\int_{0}^{s} f(v) d v
$$

for the existence of solutions to problem (1.3).

Loewner and Nirenberg [24] showed that if $f(u)=u^{p_{0}}$ with $p_{0}=(N+2) /(N-2), N>2$, then problem (1.3) has a unique positive solution $u$ which satisfies

$$
\lim _{d(x) \rightarrow 0} u(x)(d(x))^{(N-2) / 2}=(N(N-2) / 4)^{(N-2) / 4} .
$$

When $f$ satisfies $\left(\mathrm{f}_{1}\right),\left(\mathrm{f}_{3}\right)$, and the condition that

$\left(\mathrm{f}_{4}\right)$ there exist $p>1, S_{0}>0$ such that $f(s) / s^{p}$ is increasing on $\left[S_{0}, \infty\right)$

and $b \in C^{\alpha}(\Omega)$ which is positive in $\Omega$ and satisfies

$\left(\mathrm{b}_{01}\right)$ there exist $b_{0}>0$ and $\sigma \in(0,2)$ such that

$$
\lim _{d(x) \rightarrow 0} b(x)(d(x))^{\sigma}=b_{0},
$$


García-Melián [18] showed (by using nonlinear transformations, a perturbation method, and a comparison principle) that:

(i) if $C_{f}>1$, then for any solution $u$ of problem (1.1)

$$
\lim _{d(x) \rightarrow 0} \frac{u(x)}{\psi\left(A(d(x))^{2-\sigma}\right)}=1
$$

where

$$
A=\frac{b_{0}}{(2-\sigma)\left((2-\sigma)\left(C_{f}-1\right)+1\right)}
$$

and $\psi$ satisfies

$$
\int_{\psi(t)}^{\infty} \frac{d s}{f(s)}=t, \quad \forall t>0
$$

(ii) if $C_{f}=1$ and $h(t):=t f^{\prime}(\psi(t)) \geq 1$ for sufficiently small $t>0$, then (i) still holds.

Now we introduce a class of functions.

Let $\Lambda$ denote the set of all positive non-decreasing functions $k \in C^{1}(0, v)$ which satisfy

$$
\lim _{t \rightarrow 0^{+}} \frac{d}{d t}\left(\frac{K(t)}{k(t)}\right)=C_{k}, \quad \text { where } K(t)=\int_{0}^{t} k(s) d s
$$

We note that for each $k \in \Lambda$,

$$
\lim _{t \rightarrow 0^{+}} \frac{K(t)}{k(t)}=0 \quad \text { and } \quad C_{k} \in[0,1]
$$

The set $\Lambda$ was first introduced by Cîrstea and Rădulescu. Meanwhile, Cîrstea and Rădulescu [12-15] introduced the Karamata regular variation theory to study the boundary behavior and uniqueness of solutions for problem (1.3) and obtained a series of rich and significant information about the boundary behavior of the blow-up solutions.

Inspired by the above works, in this paper, by constructing new comparison functions, we consider the exact asymptotic behavior of the solution $u$ of problem (1.1) near $\partial \Omega$ under appropriate conditions on $b(x)$.

Suppose $b$ also satisfies

$\left(\mathrm{b}_{2}\right)$ there exist some $k \in \Lambda$ and a positive constant $b_{0} \in \mathbb{R}$ such that

$$
\lim _{d(x) \rightarrow 0} \frac{b(x)}{k^{4}(d(x))}=b_{0}
$$

The key to our estimates in this paper is the solution to the problem

$$
\int_{\phi(t)}^{\infty} \frac{d s}{(f(s))^{\frac{1}{3}}}=t, \quad t>0
$$

Our main results are summarized as follows. 
Theorem 1.1 Let $f$ satisfy $\left(\mathrm{f}_{1}\right)-\left(\mathrm{f}_{3}\right)$, satisfy $\left(\mathrm{b}_{1}\right)-\left(\mathrm{b}_{2}\right)$ and $1 \leq C_{f}<\frac{3}{2}$. Then, for any solution u of problem (1.1),

$$
\lim _{d(x) \rightarrow 0} \frac{u(x)}{\phi\left(K^{\frac{4}{3}}(d(x))\right)}=\xi_{0},
$$

where $\phi$ is uniquely determined by (1.8) and

$$
\xi_{0}=\left(\left(\frac{4}{3}\right)^{3} \frac{C_{k}}{b_{0}}+\left(\frac{4}{3}\right)^{4} \frac{5 C_{f}-3}{b_{0}\left(3-2 C_{f}\right)}\right)^{\frac{C_{f}-1}{3-2 C_{f}}} .
$$

In particular, when $C_{f}=1$, $u$ verifies

$$
\lim _{d(x) \rightarrow 0} \frac{u(x)}{\phi\left(K^{\frac{4}{3}}(d(x))\right)}=1 .
$$

Remark 1.1 For the existence of solutions for problem (1.1), see A. Mohammed and S. Mohammed $[9,10]$.

The outline of this paper is as follows. In Sections 2 and 3, we give some preparation that will be used in the next section. The proof of Theorem 1.1 will be given in Section 4 .

\section{Preparation}

Our approach relies on Karamata regular variation theory established by Karamata in 1930 which is a basic tool in the theory of stochastic process (see [29-31] and the references therein). In this section, we first give a brief account of the definition and properties of regularly varying functions involved in our paper (see [29-31]).

Definition 2.1 A positive measurable function $f$ defined on $[a, \infty)$, for some $a>0$, is called regularly varying at infinity with index $\rho$, written as $f \in R V_{\rho}$, if for each $\xi>0$ and some $\rho \in \mathbb{R}$,

$$
\lim _{s \rightarrow \infty} \frac{f(\xi s)}{f(s)}=\xi^{\rho} .
$$

In particular, when $\rho=0, f$ is called slowly varying at infinity.

Clearly, if $f \in R V_{\rho}$, then $L(s):=f(s) / s^{\rho}$ is slowly varying at infinity.

Definition 2.2 A positive measurable function $f$ defined on $[a, \infty)$, for some $a>0$, is called rapidly varying at infinity if for each $\rho>1$

$$
\lim _{s \rightarrow \infty} \frac{f(s)}{s^{\rho}}=\infty .
$$

Some basic examples of slowly varying functions at infinity are

(1) every measurable function on $[a, \infty)$ which has a positive limit at infinity;

(2) $(\ln s)^{\beta}$ and $(\ln (\ln s))^{\beta}, \beta \in \mathbb{R}$;

(3) $e^{(\ln s)^{p}}, 0<p<1$, 
and some basic examples of rapidly varying functions at infinity are

(1) $e^{s}$ and $e^{e^{s}}$;

(2) $e^{e^{(\ln s)^{p}}}, e^{s^{p}}$ and $e^{e^{s^{p}}}, p>0$;

(3) $s^{\beta} e^{(\ln s)^{p}}$ and $(\ln s)^{\beta} e^{(\ln s)^{p}}, p>1, \beta \in \mathbb{R}$;

(4) $(\ln s)^{\beta} e^{s^{p}}$ and $s^{\beta} e^{s^{p}}, p>0, \beta \in \mathbb{R}$.

We also see that a positive measurable function $g$ defined on $(0, a)$ for some $a>0$, is regularly varying at zero with index $\sigma$ (written as $g \in R V Z_{\sigma}$ ) if $t \rightarrow g(1 / t)$ belongs to $R V_{-\sigma}$. Similarly, $g$ is called rapidly varying at zero if $t \rightarrow g(1 / t)$ is rapidly varying at infinity.

Proposition 2.1 (Uniform convergence theorem) Iff $\in R V_{\rho}$, then (2.1) holds uniformly for $\xi \in\left[c_{1}, c_{2}\right]$ with $0<c_{1}<c_{2}$. Moreover, if $\rho<0$, then uniform convergence holds on intervals of the form $\left(a_{1}, \infty\right)$ with $a_{1}>0$; if $\rho>0$, then uniform convergence holds on intervals $\left(0, a_{1}\right]$ provided $f$ is bounded on $\left(0, a_{1}\right]$ for all $a_{1}>0$.

Proposition 2.2 (Representation theorem) A function L is slowly varying at infinity if and only if it may be written in the form

$$
L(s)=\varphi(s) \exp \left(\int_{a_{1}}^{s} \frac{y(\tau)}{\tau} d \tau\right), \quad s \geq a_{1},
$$

for some $a_{1} \geq a$, where the functions $\varphi$ and $y$ are measurable and for $s \rightarrow \infty, y(s) \rightarrow 0$, and $\varphi(s) \rightarrow c_{0}$, with $c_{0}>0$.

We call

$$
\hat{L}(s)=c_{0} \exp \left(\int_{a_{1}}^{s} \frac{y(\tau)}{\tau} d \tau\right), \quad s \geq a_{1},
$$

normalized slowly varying at infinity and

$$
f(s)=c_{0} s^{\rho} \hat{L}(s), \quad s \geq a_{1},
$$

normalized regularly varying at infinity with index $\rho$ (and written as $f \in N R V_{\rho}$ ).

Similarly, $g$ is called normalized regularly varying at zero with index $\sigma$, written as $g \in$ $N R V Z_{\sigma}$, if $t \rightarrow g(1 / t)$ belongs to $N R V_{-\sigma}$.

A function $f \in R V_{\rho}$ belongs to $N R V_{\rho}$ if and only if

$$
f \in C^{1}\left[a_{1}, \infty\right) \text { for some } a_{1}>0 \text { and } \lim _{s \rightarrow \infty} \frac{s f^{\prime}(s)}{f(s)}=\rho .
$$

Proposition 2.3 If functions $L, L_{1}$ are slowly varying at infinity, then

(i) $L^{\sigma}$ for every $\sigma \in \mathbb{R}, c_{1} L+c_{2} L_{1}\left(c_{1} \geq 0, c_{2} \geq 0\right.$ with $\left.c_{1}+c_{2}>0\right), L \circ L_{1}\left(\right.$ if $L_{1}(t) \rightarrow+\infty$ as $t \rightarrow+\infty)$, are also slowly varying at infinity.

(ii) For every $\theta>0$ and $t \rightarrow+\infty, t^{\theta} L(t) \rightarrow+\infty, t^{-\theta} L(t) \rightarrow 0$.

(iii) For $\rho \in \mathbb{R}$ and $t \rightarrow+\infty, \frac{\ln (L(t))}{\ln t} \rightarrow 0$ and $\frac{\ln \left(t^{\rho} L(t)\right)}{\ln t} \rightarrow \rho$.

\section{Proposition 2.4}

(i) If $f_{1} \in R V_{\rho_{1}}, f_{2} \in R V_{\rho_{2}}$ with $\lim _{t \rightarrow \infty} f_{2}(t)=\infty$, then $f_{1} \circ f_{2} \in R V_{\rho_{1} \rho_{2}}$.

(ii) Iff $\in R V_{\rho}$, then $f^{\alpha} \in R V_{\rho \alpha}$ for every $\alpha \in \mathbb{R}$. 
Proposition 2.5 (Asymptotic behavior) If a function $L$ is slowly varying at infinity, then for $a \geq 0$ and $t \rightarrow \infty$,

(i) $\int_{a}^{t} s^{\beta} L(s) d s \cong(\beta+1)^{-1} t^{1+\beta} L(t)$, for $\beta>-1$;

(ii) $\int_{t}^{\infty} s^{\beta} L(s) d s \cong(-\beta-1)^{-1} t^{1+\beta} L(t)$, for $\beta<-1$.

Proposition 2.6 (Asymptotic behavior) If a function $L$ is slowly varying at zero, then for $a>0$ and $t \rightarrow 0^{+}$,

(i) $\int_{0}^{t} s^{\rho} L(s) d s \cong(\rho+1)^{-1} t^{1+\rho} L(t)$, for $\rho>-1$;

(ii) $\int_{t}^{a} s^{\rho} L(s) d s \cong(-\rho-1)^{-1} t^{1+\rho} L(t)$, for $\rho<-1$.

Next, we recall here the precise definition of viscosity solutions for the problem (1.1).

Definition 2.3 A function $u \in C(\Omega)$ is a viscosity subsolution of the PDE $\Delta_{\infty} u=b(x) f(u)$ in $\Omega$ if for every $\varphi \in C^{2}(\Omega)$, with the property that $u-\varphi$ has a local maximum at some $x_{0} \in \Omega$, then

$$
\Delta_{\infty} \varphi\left(x_{0}\right) \geq b\left(x_{0}\right) f\left(u\left(x_{0}\right)\right) .
$$

Definition 2.4 A function $u \in C(\Omega)$ is a viscosity supersolution of the PDE $\Delta_{\infty} u=$ $b(x) f(u)$ in $\Omega$ if for every $\varphi \in C^{2}(\Omega)$, with the property that $u-\varphi$ has a local minimum at some $x_{0} \in \Omega$, then

$$
\Delta_{\infty} \varphi\left(x_{0}\right) \leq b\left(x_{0}\right) f\left(u\left(x_{0}\right)\right) .
$$

Definition 2.5 A function $u \in C(\Omega)$ is a viscosity solution of the PDE $\Delta_{\infty} u=b(x) f(u)$ in $\Omega$ if it is both a subsolution and a supersolution. Finally, by a solution of (1.1) we mean a function $\mathrm{u}$ that is a solution of the PDE $\Delta_{\infty} u=b(x) f(u)$ such that $u=\infty$ on $\partial \Omega$.

\section{Some auxiliary results}

In this section, we collect some useful results that will be used in the proof of the theorem.

Lemma 3.1 Let $k \in \Lambda$. Then

(i) $\lim _{t \rightarrow 0^{+}} \frac{K(t)}{k(t)}=0, \lim _{t \rightarrow 0^{+}} \frac{t k(t)}{K(t)}=C_{k}^{-1}$, i.e., $K \in N R V Z_{C_{k}^{-1}}$;

(ii) $\lim _{t \rightarrow 0^{+}} \frac{t k^{\prime}(t)}{k(t)}=\frac{1-C_{k}}{C_{k}}$, i.e., $k \in N R V Z_{\left(1-C_{k}\right) / C_{k}} ; \lim _{t \rightarrow 0^{+}} \frac{K(t) k^{\prime}(t)}{k^{2}(t)}=1-C_{k}$.

Proof The proof is similar to the proof of Lemma 2.1 in [32], so we omit it.

Lemma 3.2 Iff satisfies $\left(\mathrm{f}_{1}\right)-\left(\mathrm{f}_{3}\right)$, then

(i) $C_{f} \in[1, \infty)$;

(ii) there exists $S_{0}>0$ such that $f(s) / s^{q}$ is increasing in $\left[S_{0}, \infty\right)$, where $q \in\left(1, \frac{C_{f}}{C_{f}-1}\right)$ for $C_{f}>1$ and $q \in(1, \infty)$ for $C_{f}=1$;

(iii) if $1 \leq C_{f}<\frac{3}{2}$, $f$ satisfies the Keller-Osserman condition (1.2);

(iv) $\left(\mathrm{f}_{3}\right)$ holds for $C_{f}>1$ if and only if $f \in N R V_{C_{f} /\left(C_{f}-1\right)}$;

(v) if $C_{f}=1, f$ is rapidly varying at infinity.

Proof We only need to prove (iii). The proof of the rest can be found in [27]. 
When $C_{f}=1$, according to (ii) and Definition 2.2, we obtain for each $q>1$

$$
\lim _{s \rightarrow \infty} \frac{f(s)}{s^{q}}=\infty
$$

When $1<C_{f}<\frac{3}{2}$, by (ii) and Proposition 2.3, we obtain for each $3<q<\frac{C_{f}}{C_{f}-1}$,

$$
\lim _{s \rightarrow \infty} \frac{f(s)}{s^{q}}=\infty
$$

Consequently, there exist $S_{1}>0$ and $c_{q}>0$ such that

$$
\frac{f(s)}{s^{q}}>c_{q}, \quad \forall s \geq S_{1} .
$$

Then there exists $S_{2}>S_{1}$ such that

$$
F(s) \geq c_{q} s^{q+1}, \quad \forall s \geq S_{2}
$$

i.e., (iii) holds.

Lemma 3.3 Suppose $f$ satisfy $\left(\mathrm{f}_{1}\right)-\left(\mathrm{f}_{3}\right)$ and $1 \leq C_{f}<\frac{3}{2}$. Let $\phi$ be the solution to the problem

$$
\int_{\phi(t)}^{\infty} \frac{d s}{(f(s))^{\frac{1}{3}}}=t, \quad \forall t>0
$$

Then

(i) $-\phi^{\prime}(t)=(f(\phi(t)))^{\frac{1}{3}}, \phi(t)>0, t>0, \phi(0):=\lim _{t \rightarrow 0^{+}} \phi(t)=+\infty$ and $\phi^{\prime \prime}(t)=\frac{1}{3}(f(\phi(t)))^{-\frac{1}{3}} f^{\prime}(\phi(t)), t>0 ;$

(ii) $\phi \in N R V Z_{-\frac{3\left(C_{f}-1\right)}{3-2 C_{f}}}$;

(iii) $\phi^{\prime} \in N R V Z_{-\frac{3 C_{f}}{3-2 C_{f}}}$;

(iv) $\lim _{t \rightarrow 0^{+}} \frac{\ln (\phi(t))}{-\ln t}=\frac{3\left(C_{f}-1\right)}{3-2 C_{f}}$ and $\lim _{t \rightarrow 0^{+}} \frac{\ln (f(\phi(t)))}{-\ln t}=\frac{3 C_{f}}{3-2 C_{f}}$.

Proof By the definition of $\phi$ and a direct calculation, we show that (i) holds.

(ii) It follows from the proof of Lemma 3.1 and Proposition 2.4 (ii) that $f^{-\frac{1}{3}} \in R V \frac{C_{f}}{-\frac{3\left(C_{f}-1\right)}{}}$. Define $L_{1}(t):=f^{-\frac{1}{3}}(t) / t^{-\frac{C_{f}}{3\left(C_{f}-1\right)}}$. Then $L_{1}$ is slowly varying, and $-\frac{C_{f}}{3\left(C_{f}-1\right)}<-1$ due to $1 \leq C_{f}<$ $\frac{3}{2}$. Therefore, by Proposition 2.5, we have

$$
\lim _{t \rightarrow \infty} \frac{t f^{-\frac{1}{3}}(t)}{\int_{t}^{\infty} f^{-\frac{1}{3}}(s) d s}=\lim _{t \rightarrow \infty} \frac{t L_{1}(t) t^{-\frac{C_{f}}{3\left(C_{f}-1\right)}}}{\int_{t}^{\infty} L_{1}(s) s^{-\frac{C_{f}}{3\left(C_{f}-1\right)}} d s}=\frac{3-2 C_{f}}{3\left(C_{f}-1\right)}
$$

Hence,

$$
\lim _{t \rightarrow 0^{+}} \frac{t \phi^{\prime}(t)}{\phi(t)}=-\lim _{t \rightarrow 0^{+}} \frac{t(f(\phi(t)))^{\frac{1}{3}}}{\phi(t)}=-\lim _{s \rightarrow+\infty} \frac{(f(s))^{\frac{1}{3}} \int_{s}^{\infty} \frac{d v}{(f(v))^{\frac{1}{3}}}}{s}=-\frac{3\left(C_{f}-1\right)}{3-2 C_{f}},
$$

i.e., $\phi \in N R V Z_{-\frac{3\left(C_{f}-1\right)}{3-2 C_{f}}}$. 
(iii) By Lemma 3.2, (i) and (3.1), we know

$$
\begin{aligned}
\lim _{t \rightarrow 0^{+}} \frac{t \phi^{\prime \prime}(t)}{\phi^{\prime}(t)} & =-\frac{1}{3} \lim _{t \rightarrow 0^{+}} \frac{f^{\prime}(\phi(t)) \int_{\phi(t)}^{\infty}(f(s))^{-\frac{1}{3}} d s}{(f(\phi(t)))^{\frac{2}{3}}} \\
& =-\lim _{s \rightarrow+\infty} \frac{f^{\prime}(s) \int_{s}^{\infty}(f(v))^{-\frac{1}{3}} d v}{3(f(s))^{\frac{2}{3}}}=-\frac{1}{3} \lim _{s \rightarrow+\infty} \frac{s f^{\prime}(s)}{f(s)} \frac{\int_{s}^{\infty}(f(v))^{-\frac{1}{3}} d v}{s(f(s))^{-\frac{1}{3}}} \\
& =-\frac{3 C_{f}}{3-2 C_{f}}
\end{aligned}
$$

The last result (iv) follows from (ii)-(iii) and Proposition 2.3(iii).

\section{Proof of the theorem}

In this section, we prove Theorem 1.1.

First, we need the following result.

Lemma 4.1 (The comparison principle) ([10], Lemma 2.5) Let b satisfy $\left(\mathrm{b}_{1}\right)$, andf satisfy $\left(\mathrm{f}_{1}\right)$. Suppose $u, v \in C(\bar{\Omega})$ such that

$$
\Delta_{\infty} u \geq b(x) f(u) \quad \text { in } \Omega \text { and } \quad \Delta_{\infty} v \leq b(x) f(v) \text { in } \Omega
$$

in the viscosity sense. If $u \leq v$ on $\partial \Omega$ and $0 \leq v$ on $\partial \Omega$, then $u \leq v$ in $\Omega$.

For any $\delta>0$, we define

$$
\Omega_{\delta}=\{x \in \Omega: d(x)<\delta\}
$$

Since $\Omega$ is smooth, there exists $\delta_{0}>0$ such that $d \in C^{2}\left(\Omega_{\delta_{0}}\right)$ and $|\nabla d(x)|=1, \forall x \in \Omega_{\delta_{0}}$, and consequently $\Delta_{\infty} d=0$ in $\Omega_{\delta_{0}}$ in the viscosity sense.

Proof of Theorem 1.1 Fix a small $\varepsilon>0$. Let $\delta \in\left(0, \frac{\delta_{0}}{2}\right), \rho \in(0, \delta)$, and, for simplicity, denote

$$
d^{-}(x):=d(x)-\rho, \quad d^{+}(x):=d(x)+\rho .
$$

Define $\bar{u}_{\varepsilon}=\left(\xi_{0}+\varepsilon\right) \phi\left(K^{\frac{4}{3}}\left(d^{-}(x)\right)\right)$ for any $x \in \Omega_{2 \delta} \backslash \bar{\Omega}_{\rho}=: \Omega_{\rho}^{-}$and $\underline{u}_{\varepsilon}=\left(\xi_{0}-\varepsilon\right) \phi\left(K^{\frac{4}{3}}\left(d^{+}(x)\right)\right)$ for any $x \in \Omega_{2 \delta-\rho}=: \Omega_{\rho}^{+}$.

Let

$$
\eta(t)=\left(\xi_{0}+\varepsilon\right) \phi\left(K^{\frac{4}{3}}(t)\right), \quad t \in(\rho, 2 \delta)
$$

Note that $K$ and $\phi$ are increasing and decreasing in their respective definition domains. Therefore, when $\delta$ is small enough, $\eta$ is decreasing in $(\rho, 2 \delta)$. Let $\zeta$ be the inverse of $\eta$. One can easily check that

$$
\zeta^{\prime}(t)=\frac{1}{\eta^{\prime}(\zeta(t))}=\left(\frac{4}{3}\left(\xi_{0}+\varepsilon\right) \phi^{\prime}\left(K^{\frac{4}{3}}(\zeta(t))\right) K^{\frac{1}{3}}(\zeta(t)) k(\zeta(t))\right)^{-1}
$$


and

$$
\begin{aligned}
\zeta^{\prime \prime}(t)= & -\left(\frac{4}{3}\left(\xi_{0}+\varepsilon\right) \phi^{\prime}\left(K^{\frac{4}{3}}(\zeta(t))\right) K^{\frac{1}{3}}(\zeta(t)) k(\zeta(t))\right)^{-3} \\
& \times\left(\frac{16}{9}\left(\xi_{0}+\varepsilon\right) \phi^{\prime \prime}\left(K^{\frac{4}{3}}(\zeta(t))\right) K^{\frac{2}{3}}(\zeta(t)) k^{2}(\zeta(t))\right. \\
& +\frac{4}{9}\left(\xi_{0}+\varepsilon\right) \phi^{\prime}\left(K^{\frac{4}{3}}(\zeta(t))\right) K^{-\frac{2}{3}}(\zeta(t)) k^{2}(\zeta(t)) \\
& \left.+\frac{4}{3}\left(\xi_{0}+\varepsilon\right) \phi^{\prime}\left(K^{\frac{4}{3}}(\zeta(t))\right) K^{\frac{1}{3}}(\zeta(t)) k^{\prime}(\zeta(t))\right)
\end{aligned}
$$

Let $\left(x_{0}, \psi\right) \in \Omega_{\rho}^{-} \times C^{2}\left(\Omega_{\rho}^{-}\right)$be a pair such that $\bar{u}_{\varepsilon} \geq \psi$ in a neighborhood $N$ of $x_{0}$ and $\bar{u}_{\varepsilon}\left(x_{0}\right)=\psi\left(x_{0}\right)$ Then $\varphi=\zeta(\psi) \in C^{2}\left(\Omega_{\rho}^{-}\right)$, and

$$
d^{-}(x) \leq \varphi(x) \quad \text { in } N, \quad d^{-}\left(x_{0}\right)=\varphi\left(x_{0}\right) .
$$

Since $\Delta_{\infty} d=0$ in $\Omega_{\rho}^{-}$, we have $\Delta_{\infty} \varphi\left(x_{0}\right) \geq 0$. A simple computation shows that

$$
\Delta_{\infty} \varphi=\zeta^{\prime \prime}(\psi)\left(\zeta^{\prime}(\psi)\right)^{2}|D \psi|^{4}+\left(\zeta^{\prime}(\psi)\right)^{3} \Delta_{\infty} \psi
$$

It follows by $\Delta_{\infty} \varphi\left(x_{0}\right) \geq 0$ and $\zeta^{\prime}<0$ that

$$
\Delta_{\infty} \psi\left(x_{0}\right) \leq-\zeta^{\prime \prime}\left(\psi\left(x_{0}\right)\right)\left(\zeta^{\prime}\left(\psi\left(x_{0}\right)\right)\right)^{-1}\left|D \psi\left(x_{0}\right)\right|^{4}
$$

Moreover, since $|D d(x)|=1$ for $x \in \Omega_{\rho}^{-}$and $d^{-}-\varphi$ attains a local maximum at $x_{0}$, it follows that

$$
\left|D d^{-}\left(x_{0}\right)\right|=\left|\zeta^{\prime}\left(\psi\left(x_{0}\right)\right) D \psi\left(x_{0}\right)\right|
$$

Hence

$$
\Delta_{\infty} \psi\left(x_{0}\right) \leq-\zeta^{\prime \prime}\left(\psi\left(x_{0}\right)\right)\left(\zeta^{\prime}\left(\psi\left(x_{0}\right)\right)\right)^{-5}
$$

Combining with (4.1) and (4.2), we further obtain

$$
\begin{aligned}
\Delta_{\infty} \psi\left(x_{0}\right) \leq & \left(\frac{4}{3}\left(\xi_{0}+\varepsilon\right)\right)^{3}\left(\phi^{\prime}\left(K^{\frac{4}{3}}\left(\varphi\left(x_{0}\right)\right)\right)\right)^{3} k^{4}\left(\varphi\left(x_{0}\right)\right) \\
& \times\left[\frac{4}{3} \frac{\phi^{\prime \prime}\left(K^{\frac{4}{3}}\left(\varphi\left(x_{0}\right)\right)\right) K^{\frac{4}{3}}\left(\varphi\left(x_{0}\right)\right)}{\phi^{\prime}\left(K^{\frac{4}{3}}\left(\varphi\left(x_{0}\right)\right)\right)}+\frac{1}{3}+\frac{K\left(\varphi\left(x_{0}\right)\right) k^{\prime}\left(\varphi\left(x_{0}\right)\right)}{k^{2}\left(\varphi\left(x_{0}\right)\right)}\right] .
\end{aligned}
$$

Hence,

$$
\begin{aligned}
& \Delta_{\infty} \psi\left(x_{0}\right)-b\left(x_{0}\right) f\left(\bar{u}_{\varepsilon}\left(x_{0}\right)\right) \\
& \quad \leq\left(\frac{4}{3}\left(\xi_{0}+\varepsilon\right)\right)^{3}\left(-\phi^{\prime}\left(K^{\frac{4}{3}}\left(d^{-}\left(x_{0}\right)\right)\right)\right)^{3} k^{4}\left(d^{-}\left(x_{0}\right)\right)\left[-\frac{4}{3} \frac{\phi^{\prime \prime}\left(K^{\frac{4}{3}}\left(d^{-}\left(x_{0}\right)\right)\right) K^{\frac{4}{3}}\left(d^{-}\left(x_{0}\right)\right)}{\phi^{\prime}\left(K^{\frac{4}{3}}\left(d^{-}\left(x_{0}\right)\right)\right)}\right.
\end{aligned}
$$




$$
\begin{aligned}
& \left.-\frac{1}{3}-\frac{K\left(d^{-}\left(x_{0}\right)\right) k^{\prime}\left(d^{-}\left(x_{0}\right)\right)}{k^{2}\left(d^{-}\left(x_{0}\right)\right)}-\left(\frac{4}{3}\left(\xi_{0}+\varepsilon\right)\right)^{-3} \frac{b\left(x_{0}\right)}{k^{4}\left(d^{-}\left(x_{0}\right)\right)} \frac{f\left(\bar{u}_{\varepsilon}\left(x_{0}\right)\right)}{\left(-\phi^{\prime}\left(K^{\frac{4}{3}}\left(d^{-}\left(x_{0}\right)\right)\right)\right)^{3}}\right] \\
= & :\left(\frac{4}{3}\left(\xi_{0}+\varepsilon\right)\right)^{3}\left(-\phi^{\prime}\left(K^{\frac{4}{3}}\left(d^{-}\left(x_{0}\right)\right)\right)\right)^{3} k^{4}\left(d^{-}\left(x_{0}\right)\right) I\left(x_{0}\right) .
\end{aligned}
$$

Notice that $K^{\frac{4}{3}}\left(d^{-}\left(x_{0}\right)\right) \rightarrow 0$ as $\delta \rightarrow 0$ (and thereby $x_{0}$ tends to the boundary of $\Omega$ ). Then it follows from Lemmas 3.2 and 3.3 that

$$
I\left(x_{0}\right) \rightarrow C_{k}+\frac{20 C_{f}-12}{3\left(3-2 C_{f}\right)}-b_{0}\left(\frac{3}{4}\right)^{3}\left(\xi_{0}+\varepsilon\right)^{\frac{3-2 C_{f}}{C_{f}-1}} \quad \text { as } \delta \rightarrow 0 .
$$

By the choice of $\xi_{0}$, we have $I\left(x_{0}\right)<0$ provided $\delta \in\left(0, \frac{\delta_{0}}{2}\right)$ small enough. Thus

$$
\Delta_{\infty} \psi\left(x_{0}\right) \leq b\left(x_{0}\right) f\left(\bar{u}_{\varepsilon}\left(x_{0}\right)\right)
$$

i.e., $\bar{u}_{\varepsilon}$ is a supersolution of equation (1.1) in $\Omega_{\rho}^{-}$.

In a similar way, we can show that $\underline{u}_{\varepsilon}$ is a subsolution of equation (1.1) in $\Omega_{\rho}^{+}$.

Now let $u$ be an arbitrary solution of problem (1.1). We assert that there exists a positive constant $M$ such that

$$
\begin{array}{ll}
u \leq M(\delta)+\bar{u}_{\varepsilon}, & x \in \Omega_{\rho}^{-}, \\
\underline{u}_{\varepsilon} \leq u+M(\delta), & x \in \Omega_{\rho}^{+} .
\end{array}
$$

In fact, we may choose a large $M(\delta)$ such that

$$
u \leq M(\delta)+\bar{u}_{\varepsilon} \quad \text { on } \Gamma_{2 \delta}:=\{x \in \Omega: d(x)=2 \delta\},
$$

where $M(\delta):=\max \{u(x): d(x) \geq 2 \delta\}$.

By $\left(f_{1}\right)$, we see that $\bar{u}_{\varepsilon}+M(\delta)$ is also a supersolution of equation (1.1) in $\Omega_{\rho}^{-}$. Since $u<\bar{u}_{\varepsilon}$ on $\Gamma_{\rho}:=\{x \in \Omega: d(x)=\rho\}$, (4.3) follows by Lemma 4.1.

In a similar way, we can show (4.4).

Hence, $x \in \Omega_{\rho}^{-} \cap \Omega_{\rho}^{+}$, by letting $\rho \rightarrow 0$, we have

$$
\xi_{0}-\varepsilon-\frac{M(\delta)}{\phi\left(K^{\frac{4}{3}}(d(x))\right)} \leq \frac{u(x)}{\phi\left(K^{\frac{4}{3}}(d(x))\right)}
$$

and

$$
\frac{u(x)}{\phi\left(K^{\frac{4}{3}}(d(x))\right)} \leq \xi_{0}+\varepsilon+\frac{M(\delta)}{\phi\left(K^{\frac{4}{3}}(d(x))\right)} .
$$

Moreover, by Lemma 3.3, we obtain

$$
\xi_{0}-\varepsilon \leq \liminf _{d(x) \rightarrow 0} \frac{u(x)}{\phi\left(K^{\frac{4}{3}}(d(x))\right)} \quad \text { and } \quad \limsup _{d(x) \rightarrow 0} \frac{u(x)}{\phi\left(K^{\frac{4}{3}}(d(x))\right)} \leq \xi_{0}+\varepsilon .
$$

Thus the proof is finished by letting $\varepsilon \rightarrow 0$. 


\section{Appendix}

The following result shows that $\phi$ defined by (1.8) is stable.

Lemma A.1 Let $f_{1}$ and $f_{2}$ be positive continuous on $(0, \infty)$ and $f_{1} \in R V_{\rho+3}(\rho>0)$. If

$$
\lim _{t \rightarrow \infty} \frac{f_{1}(t)}{f_{2}(t)}=1 \quad \text { and } \quad \int_{\phi_{1}(t)}^{\infty} \frac{d s}{\left(f_{1}(s)\right)^{\frac{1}{3}}}=t=\int_{\phi_{2}(t)}^{\infty} \frac{d s}{\left(f_{1}(s)\right)^{\frac{1}{3}}}, \quad \forall t>0
$$

then

$$
\lim _{t \rightarrow 0^{+}} \frac{\phi_{1}(t)}{\phi_{2}(t)}=1
$$

Proof Since $f_{1} \in R V_{\rho+3}(\rho>0), f_{1}^{\frac{1}{3}} \in R V_{\frac{\rho}{3}+1}$. Hence, for any given small $\varepsilon>0$, there exists $t_{1}>0$ such that

$$
\left(f_{1}((1+\varepsilon) s)\right)^{\frac{1}{3}}>(1+\varepsilon)^{\frac{\rho}{6}+1}\left(f_{1}(s)\right)^{\frac{1}{3}}
$$

and

$$
\left(f_{2}(s)\right)^{\frac{1}{3}}<(1+\varepsilon)^{\frac{\rho}{6}}\left(f_{1}(s)\right)^{\frac{1}{3}}, \quad \forall s>\phi_{2}(t), \forall t \in\left(0, t_{1}\right) .
$$

It follows that, for $t \in\left(0, t_{1}\right)$,

$$
\begin{aligned}
\int_{\phi_{1}(t)}^{\infty} \frac{d s}{\left(f_{1}(s)\right)^{\frac{1}{3}}} & =\int_{\phi_{2}(t)}^{\infty} \frac{d s}{\left(f_{2}(s)\right)^{\frac{1}{3}}} \\
& >\int_{\phi_{2}(t)}^{\infty} \frac{d s}{(1+\varepsilon)^{\frac{\rho}{6}}\left(f_{1}(s)\right)^{\frac{1}{3}}}>\int_{\phi_{2}(t)}^{\infty} \frac{(1+\varepsilon) d s}{\left(f_{1}((1+\varepsilon) s)\right)^{\frac{1}{3}}} \\
& =\int_{(1+\varepsilon) \phi_{2}(t)}^{\infty} \frac{d s}{\left(f_{1}(s)\right)^{\frac{1}{3}}} .
\end{aligned}
$$

This implies that

$$
\phi_{1}(t)<(1+\varepsilon) \phi_{2}(t), \quad t \in\left(0, t_{1}\right) .
$$

Similarly we can show that there exists $t_{2}>0$ such that

$$
\phi_{2}(t)<(1+\varepsilon) \phi_{1}(t), \quad t \in\left(0, t_{2}\right) .
$$

Therefore

$$
\lim _{t \rightarrow 0^{+}} \frac{\phi_{1}(t)}{\phi_{2}(t)}=1
$$

The proof is finished. 


\section{Acknowledgements}

The author is thankful to the honorable reviewers for their valuable suggestions and comments, which improved the paper. This work was partially supported by NSF of China (Grant no. 11301250) and NSF of Shandong Province (Grant no. ZR2013AQ004), the Applied Mathematics Enhancement Program (AMEP) of Linyi University (Grant no. 2013001) and PhD research startup foundation of Linyi University (Grant no. LYDX2013BS049).

\section{Received: 25 August 2015 Accepted: 10 November 2015 Published online: 25 November 2015}

\section{References}

1. Aronsson, G: Extension of functions satisfying Lipschitz conditions. Ark. Mat. 6, 551-561 (1967)

2. Crandall, M, Lions, PL: Viscosity solutions and Hamilton-Jacobi equations. Trans. Am. Math. Soc. 277, 1-42 (1983)

3. Crandall, M, Evans, LC, Lions, PL: Some properties of viscosity solutions of Hamilton-Jacobi equations. Trans. Am. Math. Soc. 282, 487-502 (1984)

4. Crandall, M, Ishii, H, Lions, PL: User's guide to viscosity solutions of second order partial differential equations. Bull. Am. Math. Soc. 27, 1-67 (1992)

5. Jensen, R: Uniqueness of Lipschitz extensions: minimizing the sup norm of the gradient. Arch. Ration. Mech. Anal. 123(1), 51-74 (1993)

6. Aronson, G, Crandall, MG, Juutinen, P: A tour of the theory of absolute minimizing functions. Bull. Am. Math. Soc. 41 439-505 (2004)

7. Crandall, MG: A visit with the 1-Laplace equation. In: Calculus of Variations and Nonlinear Partial Differential Equations. Lecture Notes in Math., vol. 1927, pp. 75-122. Springer, Berlin (2008)

8. Juutinen, P, Rossi, J: Large solutions for the infinity Laplacian. Adv. Calc. Var. 1, 271-289 (2008)

9. Mohammed, A, Mohammed, S: Boundary blow-up solutions to degenerate elliptic equations with non-monotone inhomogeneous terms. Nonlinear Anal. 75, 3249-3261 (2012)

10. Mohammed, A, Mohammed, S: On boundary blow-up solutions to equations involving the $\infty$-Laplacian. Nonlinear Anal. 74, 5238-5252 (2011)

11. Bandle, C, Marcus, M: Large solutions of semilinear elliptic equations: existence, uniqueness and asymptotic behavior. J. Anal. Math. 58, 9-24 (1992)

12. Cîrstea, F, Rădulescu, V: Uniqueness of the blow-up boundary solution of logistic equations with absorbtion. C. R. Math. Acad. Sci. Paris, Sér. I 335, 447-452 (2002)

13. Cîrstea, F, Rădulescu, V: Asymptotics for the blow-up boundary solution of the logistic equation with absorption. C. R Math. Acad. Sci. Paris, Sér. I 336, 231-236 (2003)

14. Cîrstea, F, Rǎdulescu, V: Nonlinear problems with boundary blow-up: a Karamata regular variation theory approach Asymptot. Anal. 46, 275-298 (2006)

15. Cîrstea, F: Elliptic equations with competing rapidly varying nonlinearities and boundary blow-up. Adv. Differ. Equ. $12,995-1030$ (2007)

16. Du, Y, Huang, Q: Blow-up solutions for a class of semilinear elliptic and parabolic equations. SIAM J. Math. Anal. 31 , 1-18 (1999)

17. García-Melián, J, Letelier Albornoz, R, Sabina de Lis, J: Uniqueness and asymptotic behaviour for solutions of semilinear problems with boundary blow-up. Proc. Am. Math. Soc. 129(12), 3593-3602 (2001)

18. García-Melián, J: Boundary behavior of large solutions to elliptic equations with singular weights. Nonlinear Anal. 67, 818-826 (2007)

19. García-Melián, J: Uniqueness of positive solutions for a boundary blow-up problem. J. Math. Anal. Appl. 360(2), 530-536 (2009)

20. Juutinen, P: Convexity of solutions to boundary blow-up problems. Commun. Pure Appl. Anal. 12, $2267-2275$ (2013)

21. Keller, JB: On solutions of $\Delta u=f(u)$. Commun. Pure Appl. Math. 10, 503-510 (1957)

22. Lair, AV: A necessary and sufficient condition for existence of large solutions to semilinear elliptic equations. J. Math. Anal. Appl. 240, 205-218 (1999)

23. Lazer, AC, McKenna, PJ: Asymptotic behavior of solutions of boundary blowup problems. Differ. Integral Equ. 7 1001-1019 (1994)

24. Loewner, C, Nirenberg, L: Partial differential equations invariant under conformal or projective transformations. In: Contributions to Analysis (a collection of papers dedicated to Lipman Bers), pp. 245-272. Academic Press, New York (1974)

25. López-Gómez, J: Optimal uniqueness theorems and exact blow-up rates of large solutions. J. Differ. Equ. 224, 385-439 (2006)

26. Osserman, R: On the inequality $\Delta u \geq f(u)$. Pac. J. Math. 7, 1641-1647 (1957)

27. Zhang, Z, Ma, Y, Mi, L, Li, X: Blow-up rates of large solutions for elliptic equations. J. Differ. Equ. 249, 180-199 (2010)

28. $\mathrm{Mi}$, L, Liu, B: Second order expansion for blowup solutions of semilinear elliptic problems. Nonlinear Anal. 75 2591-2613 (2012)

29. Maric, V: Regular Variation and Differential Equations. Lecture Notes in Math., vol. 1726. Springer, Berlin (2000)

30. Resnick, SI: Extreme Values, Regular Variation, and Point Processes. Springer, Berlin (1987)

31. Seneta, R: Regular Varying Functions. Lecture Notes in Math., vol. 508. Springer, Berlin (1976)

32. Zhang, Z: The second expansion of the solution for a singular elliptic boundary value problems. J. Math. Anal. Appl. $381,922-934(2011)$ 